\title{
Resolution Assessment in Electron Tomography: a Cross-Validation Approach
}

\author{
G. Cardone,* K. Grünewald,** A. C. Steven*
}

*Laboratory of Structural Biology, National Institute of Arthritis, Musculoskeletal and Skin Diseases, National Institutes of Health, Bethesda, MD 20892, USA.

**Department of Molecular Structural Biology, Max Planck Institute of Biochemistry, 82152, Martinsried, Germany.

Although resolution assessment in Single Particle Analysis (SPA) remains short of consensus, several measures - such as the Fourier Shell Correlation and the Differential Phase Residual - are widely used. FSC and DPR exploit redundancy in the data set by measuring the consistency, as a function of spatial frequency, of two density maps calculated from an arbitrary division of the data into half sets. Resolution assessment in electron tomography poses additional problems: first, there is generally only one density map; second, limits are posed by number of the projections, and anisotropy arises from the "missing wedge" effect. To date, only one criterion has been proposed for tomography, and its applicability is confined to a particular class of reconstruction algorithms [1]. In practice, resolution assessment in electron tomography has usually relied on the visibility (subjectively decided) of certain details or, if a component of known structure is present in the tomogram, it can be used as reference in an $\mathrm{FSC}$ calculation ( $\left.\mathrm{FSC}_{\text {ref }}\right)$ [2]. We now propose a criterion for the resolution of tomograms, based on cross-validation. It operates by calculating, as a function of the spatial frequency, the consistency between each projection and the corresponding reprojection of a tomogram calculated from all other projections. We call it the "leave one out" (LOO) method. As adapted to compensate for the differing noise statistics of the data compared, and averaged over all projections, we obtain the 3D Noise-compensated LOO measure (3DNLOO).

A tilt series consists of $N$ projections. $X^{(i)}$ is the Fourier transform of projection $i$. The tomogram generated from the full tilt series produces reprojections whose Fourier transforms are $\tilde{X}^{(i)}$. Tomograms generated by excluding the corresponding input projections yield reprojections with Fourier transforms, $\tilde{X}^{-(i)}$. At a given radial frequency $k$, we define the 2DNLOO for projection $i$ as

$$
2 D N L O O^{(i)}(k)=\frac{F R C_{X \tilde{X}^{-}}^{(i)}(k)}{F R C_{X \tilde{X}}^{(i)}(k)},
$$

i.e. the ratio between two Fourier Ring Correlations [3]. The difference in the noise statistics of the projection and reprojection is compensated by including, as denominator, the FRC calculated between the input projection and the corresponding reprojection of the full tomogram. According to the Central Section Theorem, 2DNLOO can be interpreted as a resolution estimate related to a slice of the 3D Fourier transform of the reconstructed volume. The 3DNLOO measure of resolution for the full reconstruction is derived from 2DNLOO, by integrating separately, both at the numerator and the denominator, over the tilt series. Since 3DNLOO is basically a (normalized) FSC coefficient, resolution is specified as the frequency at which this measure falls below a given threshold, as in SPA, where values of $0.5,0.3,3 \sigma$, and 0.142 are in use. Resolution as given by $3 \mathrm{DNLOO}$ is linked to the signal-to-noise ratio (SNR) of the tomogram by $3 D N L O O \cong S N R /(S N R+1)$. This relationship allows us to relate the threshold value used to determine resolution from the $3 \mathrm{DNLOO}$ curve to a specific SNR. The NLOO approach also 
provides a straightforward means to assess the anisotropy of resolution in a tomogram, i.e. by plotting resolution, as given by $2 \mathrm{DNLOO}$ for a specified threshold, as a function of tilt-angle.

In order to assess the consistency of $3 \mathrm{DNLOO}$ with the previously used $\mathrm{FSC}_{\text {ref, }}$ which is intuitively appealing but is not applicable unless the tomographic volume happens to contain a known reference structure, we performed the following simulation experiment. Tomograms were calculated from a tilt series that was generated computationally from a cryo-EM density map of the Hepatitis B Virus capsid, a round particle of $320 \AA$ diameter [4]. This map has a resolution of $9 \AA$ and, for present purposes, is essentially noise-free. In a first experiment, the tilt series covered the range, $-72^{\circ}$ to $+72^{\circ}$ at $1^{\circ}$ intervals, and white noise was added to the projections at a SNR of 0.5 . We compared the 3DNLOO results to those of a FSCref, calculated between the tomogram and the original density map. The two measures show generally good agreement (Figure 1a), and the tomogram resolution, at a threshold of 0.5 , is $\sim 17 \AA$ in each case. Its resolution is reduced relative to that of the original density map by the discrete angular sampling and the introduced noise. Different levels of noise were tested with a larger angular step of $2^{\circ}$ (Figure $1 \mathrm{~b}$ ) and also gave good agreement between the two measures, indicating that 3DNLOO provides reliable estimates of resolution under a variety of conditions. Preliminary applications of the method to real (experimental) sets of tomographic data, from both vitrified specimens and plastic sections, have yielded similarly encouraging results. A program implementing 3DNLOO, called ELECTRA, has been written and is available on request.

\section{References}

[1] P. A. Penczek, J. Struct. Biol. 138 (2002) 34.

[2] K. Grünewald et al., Science 302 (2003) 1396.

[3] W. O. Saxton and W. Baumeister, J. Microsc. 127 (Pt 2) (1982) 127.

[4] J. F. Conway et al., Nature 386 (1997) 91.

a)

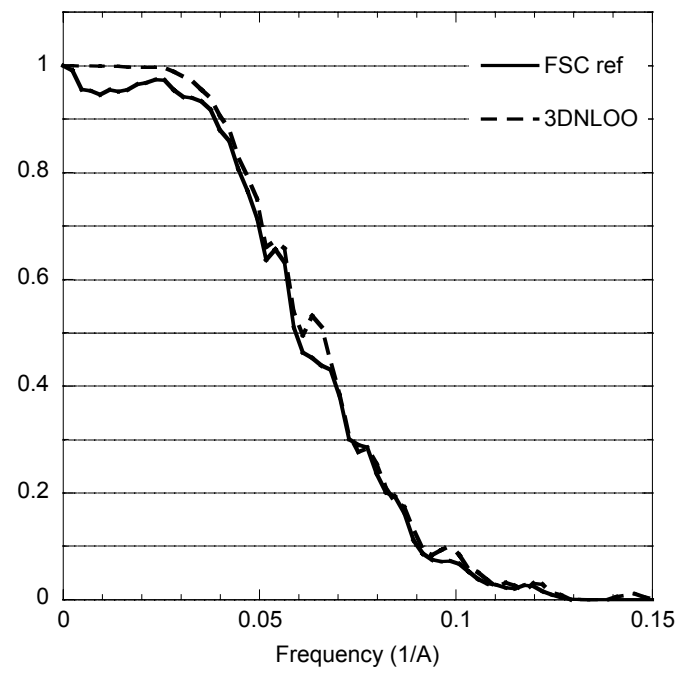

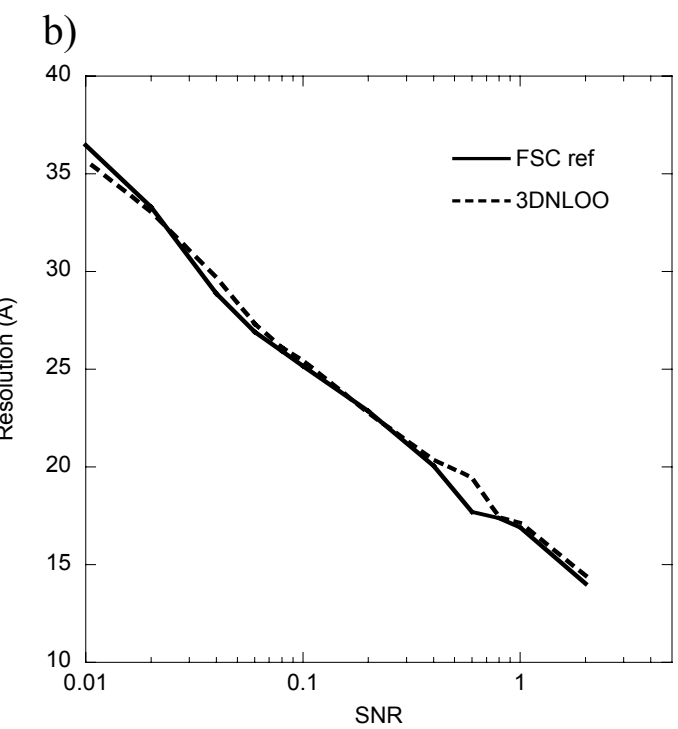

Fig. 1. Resolution curves were calculated for synthetic tomograms of the HBV capsid. a) for an angular step of $1^{\circ}$ (145 images) and $\mathrm{SNR}=0.5$. b) Resolution versus SNR for angular step of $2^{\circ}(73$ images). Threshold value $=0.5$. 